\title{
BMJ Open Validation of a clinical examination to differentiate a cervicogenic source of headache: a diagnostic prediction model using controlled diagnostic blocks
}

\author{
Scott L Getsoian (D , , ${ }^{1,2}$ Surendra M Gulati, ${ }^{1}$ Ikenna Okpareke, ${ }^{3}$ Robert J Nee, ${ }^{4}$ \\ Gwendolen A Jull ${ }^{5}$
}

To cite: Getsoian SL, Gulati SM, Okpareke I, et al. Validation of a clinical examination to differentiate a cervicogenic source of headache: a diagnostic prediction model using controlled diagnostic blocks. BMJ Open 2020;10:e035245. doi:10.1136/ bmjopen-2019-035245

- Prepublication history and additional material for this paper are available online. To view these files, please visit the journal online (http://dx.doi. org/10.1136/bmjopen-2019035245).

Received 24 October 2019 Revised 14 January 2020 Accepted 02 April 2020
Check for updates

(C) Author(s) (or their employer(s)) 2020. Re-use permitted under CC BY-NC. No commercial re-use. See rights and permissions. Published by BMJ.

For numbered affiliations see end of article.

Correspondence to Professor Scott L Getsoian; ScottGetsoian@gmail.com

\section{ABSTRACT}

Objectives Neck pain commonly accompanies recurrent headaches such as migraine, tension-type and cervicogenic headache. Neck pain may be part of the headache symptom complex or a local source. Patients commonly seek neck treatment to alleviate headache, but this is only indicated when cervical musculoskeletal dysfunction is the source of pain. Clinical presentation of reduced cervical extension, painful cervical joint dysfunction and impaired muscle function collectively has been shown to identify cervicogenic headache among patients with recurrent headaches. The pattern's validity has not been tested against the 'gold standard' of controlled diagnostic blocks. This study assessed the validity of this pattern of cervical musculoskeletal signs to identify a cervical source of headache and neck pain, against controlled diagnostic blocks, in patients with headache and neck pain.

Design Prospective concurrent validity study that employed a diagnostic model building approach to analysis.

Setting Hospital-based multidisciplinary outpatient clinic in Joliet, Illinois.

Participants A convenience sample of participants who presented to a headache clinic with recurrent headaches associated with neck pain. Sixty participants were enrolled and thirty were included in the analysis.

Outcome measures Participants underwent a clinical examination consisting of relevant tests of cervical musculoskeletal dysfunction. Controlled diagnostic blocks of C2/C3-C3/C4 established a cervical source of neck pain. Penalised logistic regression identified clinical signs to be included in a diagnostic model that best predicted participants' responses to diagnostic blocks.

Results Ten of thirty participants responded to diagnostic blocks. The full pattern of cervical musculoskeletal signs best predicted participants' responses (expected prediction error $=0.57$ ) and accounted for $65 \%$ of the variance in responses.

Conclusions This study confirmed the validity of the musculoskeletal pattern to identify a cervical source of headache and neck pain. Adopting this criterion pattern may strengthen cervicogenic headache diagnosis and inform differential diagnosis of neck pain accompanying migraine and tension-type headache.
Strengths and limitations of this study

- The validity of a pattern of cervical musculoskeletal clinical signs to identify a cervical source of headache and neck pain was tested against the 'gold standard' of controlled diagnostic cervical nerve blocks.

- Cervical musculoskeletal clinical signs were chosen a priori based on previous research that established their validity against a reference standard of clinical diagnosis of headache.

- Despite the use of penalised logistic regression to build the diagnostic prediction model, limitations of a small sample are acknowledged.

\section{INTRODUCTION}

Symptomatic overlap between recurrent headache forms of migraine, tension-type and cervicogenic headache is common and can challenge diagnosis. ${ }^{12}$ For instance, neck pain is characteristic of cervicogenic headache, ${ }^{3}$ but is also reported in up to $80 \%$ with migraine, tension-type or mixed migraine and tension-type headache cases. ${ }^{45}$ The presence of neck pain in various headache types reflects the interaction between trigeminal and cervical afferents and the bidirectional pathway in the trigeminocervical nucleus. ${ }^{6}$ The origin of neck pain in headache is variable. It may have a local cervical nociceptive source. Conversely, the neck pain may be part of the headache attack with a central cause through mechanisms of convergence and central sensitisation. ${ }^{78}$ The presence of neck pain can confound diagnosis and has fuelled debate on whether migraine is too often misdiagnosed as cervicogenic headache and vice versa. Failure to recognise the origin of neck pain can compromise the diagnosis ${ }^{9-11}$ and subsequent treatment ${ }^{12}$ of patients with recurrent headache. 
As neck pain and tenderness may reflect sensitised neck afferents rather than a local cervical nociceptive source, both the International Headache Society (ICHD-3) ${ }^{3}$ and Cervicogenic Headache International Study Group (CHISG) ${ }^{13}$ classification criteria recommend diagnostic cervical nerve or joint blocks to add to evidence for ${ }^{3}$ or confirm ${ }^{13}$ a cervical origin of neck pain and headache in cervicogenic headache. These invasive techniques are usually not feasible or suitable for initial differential diagnosis of cervicogenic headache. A clinical evaluation is the usual first-line approach. The ICHD- $3^{3}$ and CHISG $^{13}$ criteria both nominate reduced range of movement (ROM) as a clinical feature denoting a cervical cause. Reduced ROM is characteristic of a cervical disorder, ${ }^{14}$ but as a single criterion lacks diagnostic sensitivity and specificity. ${ }^{15}$ It varies within and between individuals of different age groups, ${ }^{16}$ preventing a cut-off value.

Reduced ROM is one of many physical signs of musculoskeletal disorders. A previous study found a pattern of three physical signs, not a single sign, could identify neck dysfunction associated with cervicogenic headache. ${ }^{15} 17$ The pattern was formed by the coexistence of reduced ROM (cervical extension), painful upper cervical joint dysfunction (clinical manual examination) and impaired cervical muscle function (craniocervical flexion test (CCFT) ). Clinical recognition of a cervical origin of neck pain and headache stands to strengthen clinical differential diagnostic decisions, but the validity of this pattern has not been tested in a separate group with headache, and has not been tested against the 'gold standard' of diagnostic cervical nerve or joint blocks.

This study assessed the validity of this pattern of cervical signs to detect a cervical source of headache in patients attending a neurologist for diagnosis and management of recurrent headache accompanied by neck pain. Validity was assessed by determining whether the presence of the pattern could predict a positive response to controlled diagnostic blocks of the C2/C3 and C3/C4 zygapophysial joints (third occipital nerve (TON) and medial branch blocks, respectively). These segments were chosen because blocks to higher segments pose increased risk to the patient. ${ }^{18}$ It was hypothesised that the full pattern of cervical signs would most accurately predict participants' response to C2/C3 and C3/C4 diagnostic blocks.

\section{METHODS}

This was a prospective concurrent validity study that employed a diagnostic model building approach to statistical analysis. Building a diagnostic prediction model allowed for testing both components inherent in our hypothesis. First, building a diagnostic model could determine whether all cervical signs needed to be present for optimal performance in predicting headache relief with controlled diagnostic blocks. Second, regression coefficients in the model could determine whether the direction of relationships between each cervical sign and headache relief (ie, the pattern of cervical signs) was consistent with previous research.

\section{Participants}

Participants (18-59 years) were a convenience sample, referred by a neurologist from a hospital-based outpatient headache clinic. To be included in the study, a participant had to present with recurrent headache accompanied by neck pain to test the hypothesis that, when the neck pain and headache were of cervicogenic origin, a pattern of cervical musculoskeletal signs would most accurately predict a positive response to diagnostic blocks as per ICHD- $3^{3}$ and CHISG $^{13}$ criteria. The participant could have a provisional diagnosis of either migraine, tensiontype, cervicogenic headache or mixed headache to replicate the challenge of diagnosis when neck pain is a feature of the headache. ${ }^{9-11}$ In addition, the neurologist had to consider that the neck pain warranted investigation with diagnostic blocks and the participant was willing to have the procedures. Participants were not eligible if diagnosed with other headache types, had a history of cervical fusion involving C2/C3 or C3/C4 or greater than three spinal levels, poor tolerance to lidocaine or bupivacaine, significant clotting disorder or unsafe to temporarily cease anticoagulant medication, inadequate English comprehension, or assessed by the neurologist to have significant psychological features. Eligible participants were further screened by an independent researcher using a headache questionnaire that addressed demographic information and headache history. The questionnaire incorporated ICHD-3 headache criteria ${ }^{3}$ for migraine, tension-type and cervicogenic headache, and CHISG criteria $^{13}$ for cervicogenic headache to gain standardised data across participants.

If suitable for inclusion, written and verbal information was provided, including details of the physical examination and reiteration of the diagnostic block protocol. All participants provided written informed consent.

\section{Patient and public involvement}

Patients who experience recurrent headaches were not involved in the development or implementation of this study. Patients and/or the public were not involved in the design, or conduct, or reporting, or dissemination plans of this research.

\section{Measurements and procedures \\ Clinical tests of cervical spine function}

The physical examination was conducted by an experienced physical therapist, blinded to the neurologists' headache diagnoses. The examination included the three tests for the pattern of cervical signs plus the flexion rotation test (FRT). The FRT is a movement test specific to C1/C2 dysfunction. ${ }^{19}{ }^{20}$ As we sought to identify participants with dysfunction relating to C2/3 and C3/C4, the FRT was included to help identify those with neck pain arising from a $\mathrm{C} 1 / \mathrm{C} 2$ joint dysfunction that would not 
necessarily be captured by the diagnostic blocks. ${ }^{21}$ These clinical tests were the candidate variables for building a diagnostic model that predicted headache relief with the blocks.

\section{Cervical range of motion}

Cervical extension was measured using a cervical range of motion device (CROM, Performance Attainment Associates, St. Paul, Minnesota, USA), in accordance with established procedure. ${ }^{22}$ The average of three trials was recorded. The CROM has criterion validity, ${ }^{23}$ excellent intrarater reliability, ICC $>0.85^{24}$ and standard error of measurement of $3.0^{\circ}{ }^{25}$

\section{Craniocervical flexion test}

The CCFT tests the deep neck flexor muscles, the longus colli and capitis, which are commonly impaired in cervical disorders. ${ }^{26}$ An air-filled pressure sensor (Chattanooga Stabilizer Group, Vista, California USA) behind the neck guides the participant to the test performance levels. Participants performed five increments of increasing range of craniocervical flexion to progressively increase the pressure by $2 \mathrm{~mm} \mathrm{Hg}$, from the baseline $20 \mathrm{~mm} \mathrm{Hg}$ up to $30 \mathrm{~mm} \mathrm{Hg}$, holding each stage for $10 \mathrm{~s}$. The test is scored by the pressure level which the participant achieves and holds with correct movement. The CCFT has construct validity ${ }^{26}$ intrarater reliability ICC $=0.69-0.81^{27}$ and interrater reliability $\mathrm{ICC}=0.85-0.86$.

\section{Symptomatic joint dysfunction}

Manual examination is a valid ${ }^{28}$ and reliable ${ }^{29}$ palpatory test for detecting painful upper cervical facet joint dysfunction. The examiner rated the perceived motion/ tissue compliance of each facet joint from $\mathrm{C} 0 / \mathrm{C} 1$ to $\mathrm{C} 3 /$ C4 using a scale of normal, minimal, moderate or marked restriction. ${ }^{15}$ The participant verbally rated perceived pain at each joint on a scale of 0 (no pain) to 10 (worst pain imaginable). Symptomatic joint dysfunction was present when the examiner rated motion/tissue compliance as moderate or marked restriction and the participant reported pain greater than 2/10. Participants could have joint dysfunction at one or several levels. For analysis, when joint dysfunction involved only C2/C3 and/ or $\mathrm{C} 3 / \mathrm{C} 4$ levels, or when joint dysfunction at these levels was similar to or greater than that at higher levels, the predictor variable was denoted as 'C2/C3-C3/C4 joint dysfunction'.

\section{Flexion rotation test}

Head rotation, with the neck prepositioned in full flexion, was measured with the CROM device. ${ }^{20}$ Three trials were taken to each side. The average range to the more limited side was used for analysis. The FRT has content validity, ${ }^{19}$ discriminative accuracy for cervicogenic headache arising from $\mathrm{C} 1 / \mathrm{C} 2,{ }^{21}$ intrarater reliability $\mathrm{ICC}=0.84-0.89,{ }^{20}$ and inter-rater reliability $\mathrm{ICC}=0.93$ to detect $\mathrm{C} 1 / \mathrm{C} 2$ dysfunction.

\section{Diagnostic blocks}

Controlled diagnostic blocks were administered within 4 weeks of the physical examination. Participants continued all prior medications except antiplatelet and anticoagulant medications, which were withheld prior to blocks as recommended. ${ }^{30}$ Blocks were performed at one of three outpatient clinics in Joliet, Illinois, by one of three experienced anaesthesiologists, who were board certified in anaesthesiology and pain management, and regularly performed blocks. Anaesthesiologists were blind to results of the neurology and physical examinations. To reduce false-positive rates and placebo effects, two rounds of controlled diagnostic C2/C3 and C3/C4 facet blocks were performed using lidocaine $2 \%$ and bupivacaine $0.5 \%$, respectively. ${ }^{31}$ Duration of relief was checked for correlation with duration of action of the local anaesthetic used. A successful block required that the participant reported (1) a longer relief of headache with bupivacaine than with lidocaine and (2) at least a $70 \%$ reduction in headache. This cut-off was chosen to accommodate common clinical $(50 \%)^{32}$ and more robust $(\geq 75 \%)^{33}$ standards. Blocks were performed bilaterally, unless the headache was side locked. The sequence of injections (lidocaine vs bupivacaine) was randomised. Participants used a pain diary to record hourly percentage headache relief from the blocks for up to 12 hours. The anaesthesiologist reassessed participants 2 weeks later. If they experienced at least $70 \%$ headache relief from the first round of blocks, they underwent a second procedure with the alternate anaesthetic and reassessed again 2 weeks later. Participants not reporting at least $70 \%$ relief of headache with the first blocks were categorised as nonresponders and exited the study.

\section{TON block}

The TON provides sensory innervations to the C2/C3 facet joint. ${ }^{34}$ A posterior approach was used to anaesthetise the TON at three specific locations in accord with the International Spinal Injection Society's Guidelines. ${ }^{35} 36$

Participants were positioned prone with the neck in partial flexion. The skin was prepared and draped. A C-arm fluoroscope was rotated $25^{\circ}-35^{\circ}$ caudally from the axial plane. After the articular pillars were visualised, $1 \%$ lidocaine was infiltrated into the overlying skin and subcutaneous tissues. The three target points lie on a line bisecting the C2/C3 facet joint. A 22 or 25 gauge, 3.5 inch spinal needle was inserted through the skin and advanced until seated in a plane coaxial with the X-ray path. Periodic views were obtained to ensure correct needle course. Once proper location was confirmed by imaging, $0.3 \mathrm{~mL}$ of either $2 \%$ lidocaine or $0.5 \%$ bupivacaine was injected into each of the three target points.

\section{C3/C4 medial branch block}

Following the TON block, separate needles were inserted and advanced in a coaxial technique toward the middle 'waist' of the C3 and C4 articular pillars. Once needle position was confirmed, $0.5 \mathrm{~mL}$ of the same local anaesthetic 
was injected to each of the C3 and C4 medial branch nerves to anaesthetise the $\mathrm{C} 3 / \mathrm{C} 4$ facet joint.

\section{Sample size considerations}

Logistic regression may require at least 20 participants in the limiting sample size (least frequent outcome) for each predictor being analysed to eliminate bias in regression coefficients when there is a low prevalence of binary predictors. ${ }^{37}$ The least frequent outcome was expected to be participants experiencing at least $70 \%$ headache relief from the diagnostic blocks. At least 80 participants experiencing relief from the blocks would be necessary to accommodate the four cervical signs evaluated in this study. Assuming that $21 \%$ of individuals who report headache in this study's setting have cervicogenic headache ${ }^{38} 381$ participants would be required to obtain this limiting sample size. Because it was not feasible to enroll 381 participants, we planned to employ penalised logistic regression analysis to reduce bias associated with a small limiting sample size and potentially low prevalence of binary predictors. ${ }^{37} 39$

\section{Data management and analysis}

The neurologists' diagnoses were confirmed with responses from the headache questionnaire. Headaches were classified under categories of migraine, tension-type headache, cervicogenic headache or mixed headache.

Participant data were assessed for extreme influence on the diagnostic model when Cook's distance values were over $\mathrm{D}=0.13(4 / \mathrm{n})$. Inclusion of data from participant \#19 (D=2.1) led to a model that was unstable. Furthermore, the participant's predictor data did not match that of other participants and therefore was removed from further analysis. Complete data sets were available for all other participants.

The hypothesis that the pattern of cervical signs would identify responders to $\mathrm{C} 2 / \mathrm{C} 3$ and $\mathrm{C} 3 / \mathrm{C} 4$ blocks was tested using the least absolute shrinkage and selection operator (LASSO) algorithm to build the diagnostic prediction model. LASSO reduces overfitting in small samples by applying a progressive statistical penalty that shrinks each predictor's coefficient towards zero at different rates. ${ }^{39}$ When a coefficient shrinks to zero, that predictor is eliminated from the final model. LASSO identifies an optimal model with the lowest expected prediction error that is more likely to accurately predict observations in future samples. Expected prediction errors were estimated using a 0.632 bootstrap of 1000 bootstrap samples. ${ }^{40}$ Administering LASSO within Categorical regression (CATREG) requires designation of each predictor's optimal scaling level. ${ }^{3941}$ Continuous measures of active cervical extension and the FRT were scaled as numeric, while the CCFT (ordinal) and C2/C3-C3/C4 joint dysfunction (nominal) were scaled as ordinal. These scaling decisions preserved the maximum amount of information (eg, intervals, ranks, order) from each cervical test for a thorough evaluation of whether the pattern of cervical signs accurately predicted relief from diagnostic blocks.
Explained variance, calibration and discrimination were used to assess diagnostic model performance. ${ }^{42}$ Explained variance was calculated from the apparent prediction error of the model $\left(R^{2}=1-\right.$ apparent prediction error). ${ }^{41}$ Calibration was assessed with a calibration plot that incorporated a locally weighted smoothing (LOESS) technique for binary outcomes. ${ }^{42}$ Discrimination was to be assessed with the area under a receiver operating characteristic curve (AUC). However, a zero cell in the contingency table for C2/C3-C3/C4 joint dysfunction and relief with diagnostic blocks (quasi-complete separation) prevented reporting a true AUC with a valid CI. ${ }^{43}$ Discrimination was therefore quantified by calculating the standardised mean difference (Hedges' g) between predicted probabilities for participants who did and did not experience relief with blocks. ${ }^{44}$ Data were analysed using SPSS statistical package V.25.

\section{RESULTS}

Sixty patients entered the study between August 2017 and February 2019 and 30 were included in analysis (figure 1). Participants who withdrew did so after the physical examination and prior to the diagnostic blocks, the main reason being lack of coverage by their insurance carrier. There were no statistical differences in measures of cervical dysfunction between participants who did and did not complete the study, with the exception of cervical extension (online supplementary file 1). As the aim was to test the predictors as a set, a difference in one predictor was considered acceptable.

\section{Outcomes of diagnostic blocks}

Demographics of participants, the neurologist's provisional headache classification and responses to the blocks are presented in table 1 . In 13 of 30 cases, participants were judged to have symptoms of more than one headache type and received a provisional diagnosis of a mixed headache. There were no adverse reactions to the physical examination or blocks. Ten participants $(33.3 \%)$ had at least $70 \%$ headache relief to each round of blocks, and were therefore deemed to have a cervical origin of headache (cervicogenic headache). These were likely truepositive responses because bupivacaine gave longer relief than lidocaine in all cases. Twenty participants (66.7\%) experienced less than $70 \%$ headache relief in the first round of blocks and were categorised as non-responders.

\section{Validity of the pattern of cervical signs}

The LASSO showed that all four cervical musculoskeletal signs needed to be retained for an optimal diagnostic model that had the lowest expected prediction error of 0.57 (SE 0.10) (figure 2). The LASSO also identifies the most parsimonious model that is within one SE of the expected prediction error of the optimal model. Even this most parsimonious model retained all four cervical signs (figure 2). Because LASSO regression coefficients will be biased towards zero, CATREG was reanalysed 


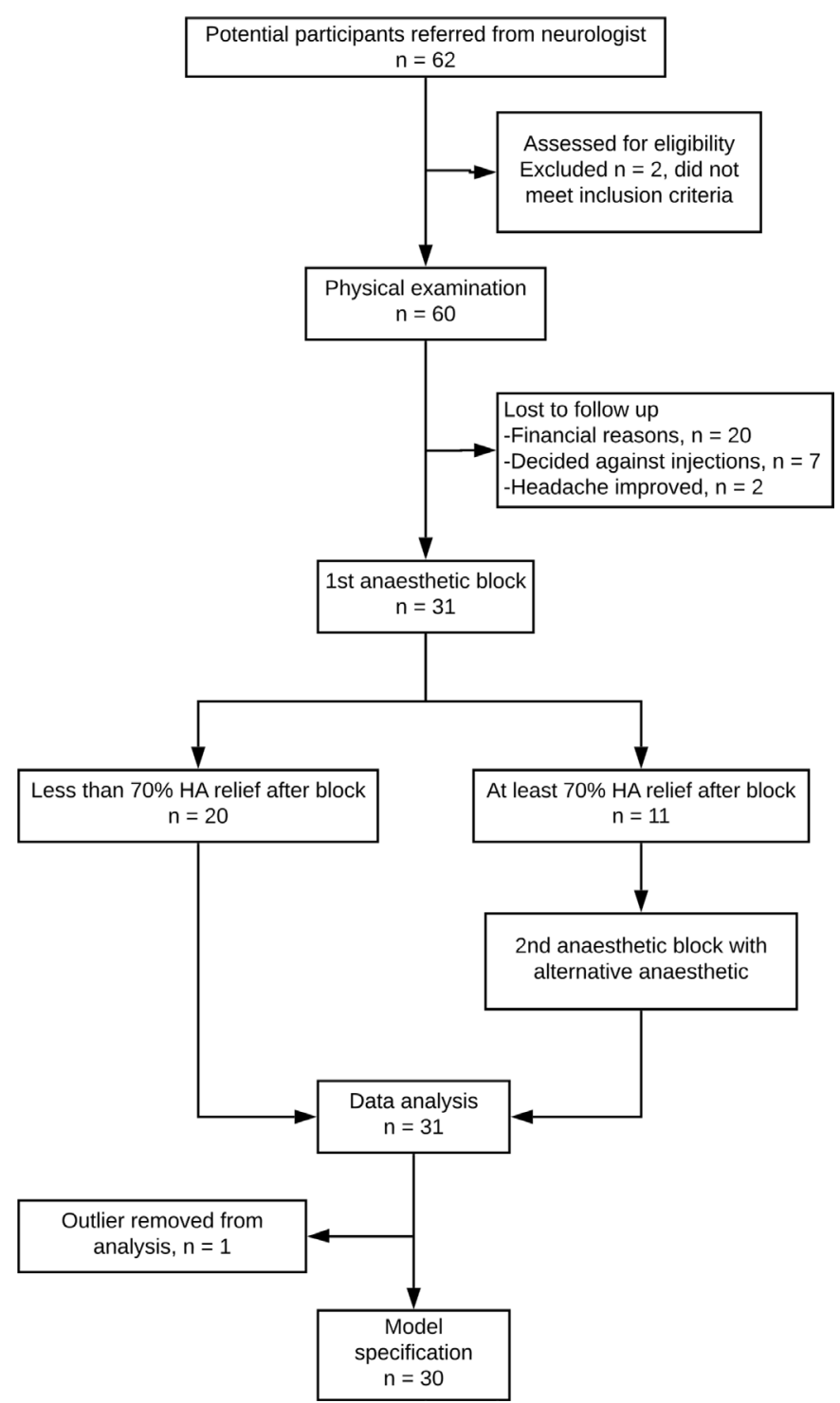

Figure 1 Participant flow diagram. HA, headache.

without the LASSO penalty to obtain less biased estimates of regression coefficients for the optimal model (table 2) ${ }^{45}$ The regression coefficients showed that the direction of relationships between each cervical sign and headache relief with blocks was consistent with previous research (table 2).

Explained variance of the optimal diagnostic model using unpenalised regression coefficients was $\mathrm{R}^{2}=0.65$ (apparent prediction error $=0.35$ ). The calibration plot suggests that predicted probabilities greater than $60 \%$ may underestimate the chance for relief with diagnostic blocks while those less than $60 \%$ may overestimate this chance (figure 3). The calibration plot also illustrated that the optimal model had perfect discrimination in our sample (figure 3) ${ }^{44}$ This is consistent with the large standardised mean difference in predicted probabilities (mean (SD)) between participants who did (0.71 (0.06)) and did not $(0.39(0.12))$ experience relief with blocks (Hedges' g=3.04). Relationships between each cervical sign and relief with blocks are reported in online supplementary files 2-5.
Table 1 Participant demographics and headache classification for responders and non-responders to the diagnostic blocks

\begin{tabular}{llll}
\hline & $\begin{array}{l}\text { Responders } \\
(\mathbf{n}=\mathbf{1 0})\end{array}$ & $\begin{array}{l}\text { Non } \\
\text { responders } \\
(\mathbf{n}=\mathbf{2 0})\end{array}$ & $\begin{array}{l}\text { Total } \\
\text { group } \\
(\mathbf{n}=\mathbf{3 0})\end{array}$ \\
\hline Age, in years (SD) & $47.0(9.3)$ & $47.7(7.9)$ & $47.4(8.2)$ \\
\hline Gender (n) & 8 & 11 & 19 \\
\hline Females & 2 & 9 & 11 \\
\hline Males & & & \\
\hline Headache diagnosis (n) & 3 & 7 & 10 \\
\hline Migraine & 1 & 3 & 4 \\
\hline Tension type & 0 & $3^{*}$ & 3 \\
\hline Cervicogenic & & & \\
\hline Mixed headaches & 0 & 2 & 2 \\
\hline Migraine + tension type & 5 & 2 & 7 \\
\hline Migraine + cervicogenic & 5 & 3 & 4 \\
\hline Tension type + cervicogenic & 1 & & \\
\hline
\end{tabular}

${ }^{*}$ One of the three participants diagnosed with cervicogenic headache likely had a C1-C2 headache not captured by the blocks because of the presence of a positive flexion rotation test, C1-C2 joint dysfunction on manual examination and impaired muscle function (craniocervical flexion test).

\section{Headache features of responders to diagnostic blocks}

Headache features of those classified with cervicogenic headache (positive response to diagnostic blocks) were reviewed to determine if there were distinguishing traits which might aid clinical differential diagnosis. The nonresponders group were heterogeneous in relation to provisional headache diagnoses which prevented any meaningful comparison as a group. The numbers in each diagnostic group were too small for any comparison (table 1) except for the migraine group where the majority were non-responders $(7 / 10)$. Table 3 presents a descriptive comparison of features of the cervicogenic responder group and the migraine non-responder group. There were no unique or major distinguishing features. The cervicogenic headache responders had more frequent headache onsets related to neck trauma/neck pain and their headaches were more often brought on by pressure to back of the head or neck. Headache changing sides was more common in the migraine group as was the reporting of premonitory symptoms.

\section{DISCUSSION}

This study confirmed the validity of a pattern of reduced movement (cervical extension), symptomatic upper cervical joint dysfunction, impaired muscle function (CCFT) and no impairment in $\mathrm{C} 1 / \mathrm{C} 2$ rotation (FRT) to predict whether participants would have substantial to full relief of pain from diagnostic blocks to the C2/C3 and C3/C4 facet joints. The optimal diagnostic model derived from the analysis required the full pattern of cervical signs to be present. These findings support 


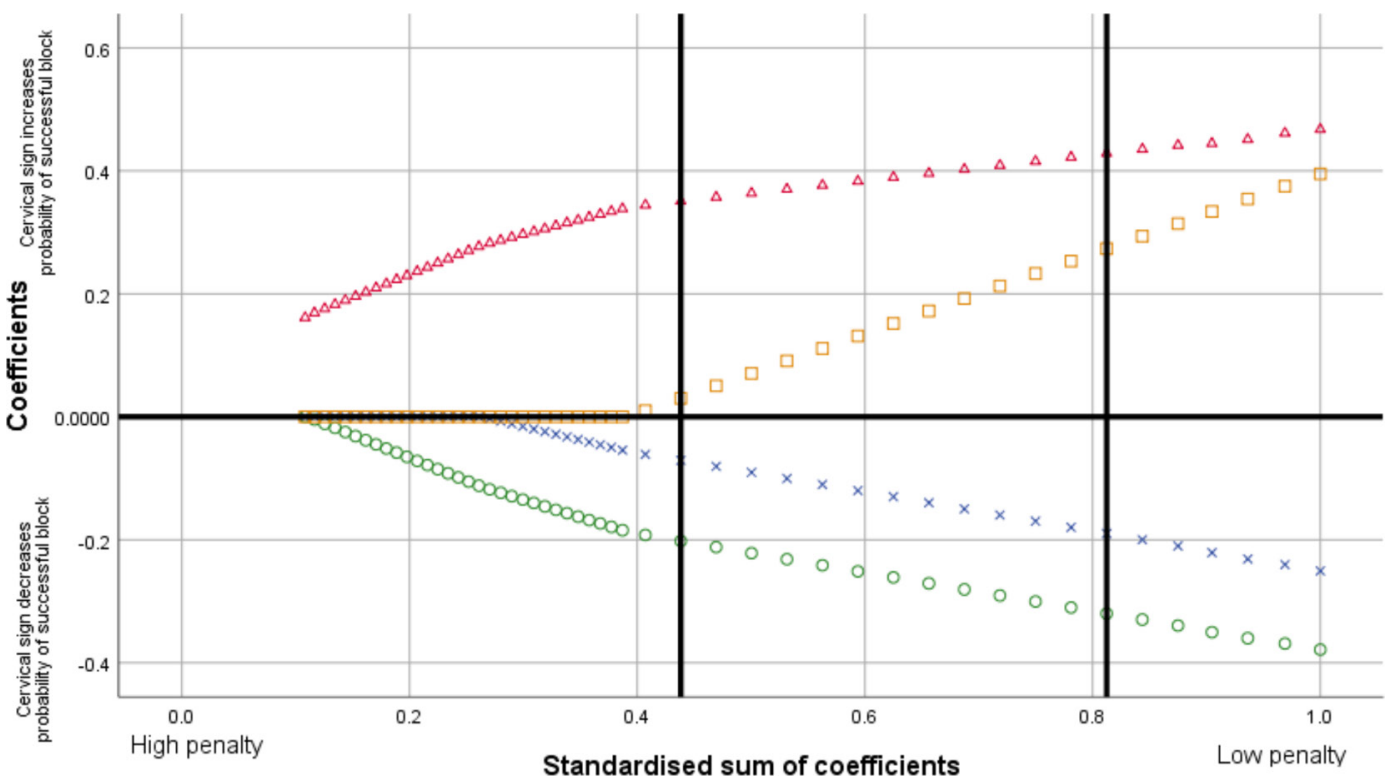

Figure 2 CATREG least absolute shrinkage and selection operator coefficient paths for cervical signs. The $x$-axis shows the weight of the applied penalty that increases from right to left and shrinks coefficients of less important predictors to zero (middle horizontal line) at a faster rate. The optimal diagnostic model (right vertical line) and the most parsimonious model (left vertical line) both retained all four cervical signs because none of the corresponding coefficients had shrunk to zero. X-->AROMext, $\Delta^{--}$ >C2/C3--C3/C4 joint dysfunction, o-->craniocervical flexion test, $\square-->$ flexion rotation test.

previous work which found the pattern had high sensitivity and specificity to identify neck dysfunction associated with cervicogenic headache. ${ }^{1517}$ This study could not account for headaches arising from $\mathrm{C} 0 / \mathrm{C} 1$ or $\mathrm{C} 1 / \mathrm{C} 2$ as diagnostic blocks to higher segments pose increased risk to the patient. ${ }^{18}$ Hence, the FRT was added to the model to account for this limitation. Review of the data suggested that at least one participant provisionally diagnosed as having cervicogenic headache and non-responsive to the blocks, had definitive C1/C2 joint dysfunction which was likely responsible for that individual's headache as it was associated with reduced extension ROM and impaired muscle function.
Current ICHD-3 and CHISG diagnostic criteria for cervicogenic headache nominate reduced ROM as the test of neck function to denote an underlying cervical musculoskeletal condition as causative of headache. ${ }^{3} 13$ While the results of this study support this criterion, they also highlight the flaw of using ROM as the only indicator for cervical musculoskeletal dysfunction in cervicogenic headache. The variability in ROM across the cohort (responders and non-responders) was substantial as was, not unexpectedly, the variation within each physical sign (online supplementary files 2-5). In other words, reduced range of cervical extension does not automatically mean a person has a cervicogenic headache. ${ }^{17}$ Likewise, 6 of

Table 2 Penalised and unpenalised coefficients for the optimal diagnostic model

\begin{tabular}{lcccc}
\hline & $\begin{array}{l}\boldsymbol{\beta} \text { Coefficient } \\
\text { (penalised) }\end{array}$ & $\begin{array}{l}\boldsymbol{\beta} \text { Coefficient } \\
\text { (unpenalised) }\end{array}$ & SE (unpenalised) $\mathbf{0 . 6 3 2 ~ b o o t s t r a p ~ ( 1 0 0 0 ) ~}$ & Sig \\
\hline AROM cervical extension & -0.190 & $-0.251^{*}$ & 0.200 & 0.222 \\
C2/C3-C3/C4 joint dysfunction & 0.429 & 0.468 & 0.228 & 0.051 \\
CCFT & -0.320 & $-0.379 \dagger$ & 0.219 & 0.069 \\
FRT & 0.274 & $0.395 \ddagger$ & 0.190 & 0.048 \\
\hline
\end{tabular}

Log odds positive block=-0.251(AROMext)+0.468(JtSignsC234) -0.379 (CCFT) +0.395(FRT).

Example calculation for a patient with impaired cervical extension $\left(20^{\circ}\right)$, presence of $\mathrm{C} 2 / \mathrm{C} 3-\mathrm{C} 3 / \mathrm{C} 4$ joint dysfunction (1), impaired deep neck flexor muscle function (CCFT $24 \mathrm{~mm} \mathrm{Hg}$ ), but minimally impaired C1/C2 motion (FRT 40 ): log odds positive

block $=-0.251(20)+0.468(1)-0.379(24)+0.395(40)$, log odds positive block=2.152, odds positive block=8.6, probability positive block=90\%.

${ }^{*}$ Negative coefficient consistent with greater impairment in cervical extension AROM (ie, smaller values) being associated with greater chance of relief with C2/C3 and C3/C4 anaesthetic blocks.

†Negative coefficient consistent with greater impairment in cervical muscle performance (ie, smaller CCFT values) being associated with greater chance of relief with $\mathrm{C} 2 / \mathrm{C} 3$ and $\mathrm{C} 3 / \mathrm{C} 4$ anaesthetic blocks.

$\ddagger$ Positive coefficient consistent with lesser impairment in C1/C2 motion/less likely C1/C2 dysfunction (ie, greater FRT values) being associated with greater chance of relief with $\mathrm{C} 2 / \mathrm{C} 3$ and $\mathrm{C} 3 / \mathrm{C} 4$ anaesthetic blocks.

CCFT, craniocervical flexion test; FRT, flexion rotation test. 


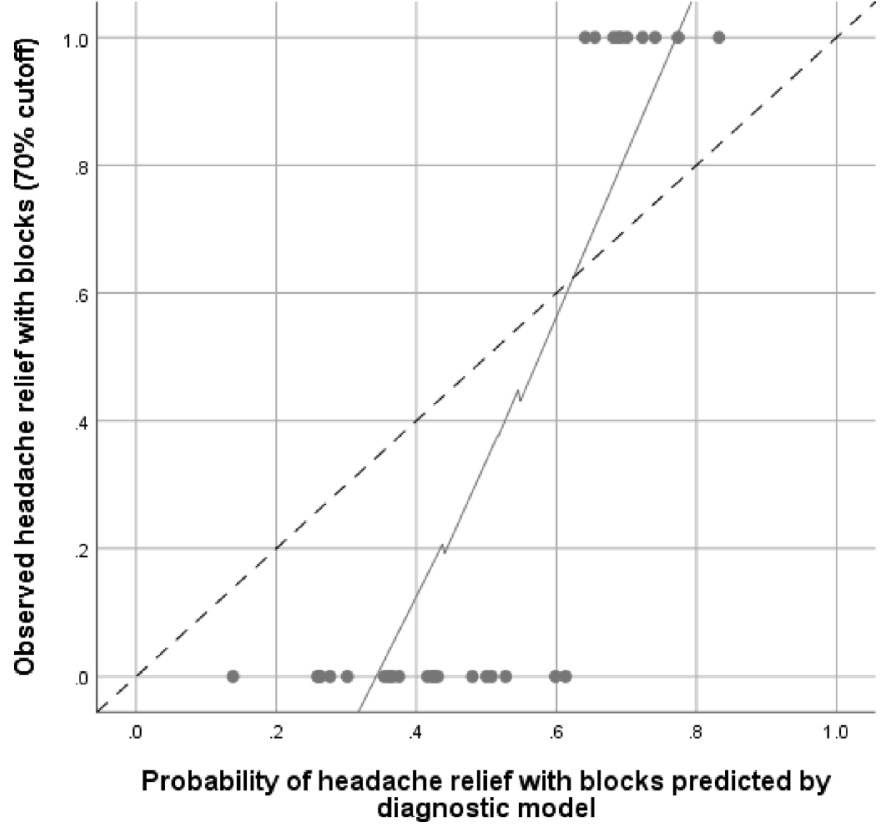

Figure 3 Calibration plot for the optimal diagnostic model using unpenalised regression coefficients. Locally weighted smoothing (LOESS) used the uniform kernel method and fitted $90 \%$ of points $(95 \% \mathrm{Cl})$. A LOESS curve of $45^{\circ}$ (dashed line) indicates perfect agreement between observed and predicted outcomes. $^{42}$

20 non-responders had pain and changes in perceived motion on manual examination of $\mathrm{C} 2 / \mathrm{C} 3$ and $\mathrm{C} 3 / \mathrm{C} 4$ (online supplementary file 3), but without other signs sufficient to fit the pattern. This latter finding could reflect increased pain sensitivity in the neck (peripherally or centrally driven hyperalgesia) associated with headache rather than related cervical musculoskeletal dysfunction. ${ }^{46}$ Cervical musculoskeletal dysfunction therefore cannot be defined with any confidence on the basis of one physical sign. It is characterised by, at the least, the simultaneous presentation of reduced cervical motion, painful upper cervical joint dysfunction and impaired muscle function, as determined in this and previous studies. ${ }^{15} 17$ This combined pattern of painfully reduced active and passive joint motion together with impaired muscle function is typical of musculoskeletal disorders as is readily observed in extremity joint conditions.

The challenges that can be experienced in differential diagnosis of recurrent headaches because of symptomatic overlap and mixed symptoms were clearly evident in this study. ${ }^{2459}$ No symptoms or combination of symptoms uniquely or clearly distinguished those with cervicogenic headache from those with migraine who were nonresponsive to the blocks (table 3 ). The three participants diagnosed as cervicogenic headache were non-responders (although one likely had a cervicogenic headache related to $\mathrm{C} 1 / \mathrm{C} 2$ dysfunction not captured by the blocks) and 3 of 10 categorised with migraine were responders to the diagnostic blocks. In this study, all participants had neck pain with their headache as an inclusion criterion. Yet only one-third received clinically relevant relief $(\geq 70 \%)$
Table 3 Comparison of headache features of participants who responded to diagnostic blocks and the nonresponders provisionally diagnosed with migraine

\begin{tabular}{|c|c|c|}
\hline Headache features & $\begin{array}{l}\text { Responders } \\
\text { cervicogenic } \\
\text { headache } \\
(n=10)\end{array}$ & $\begin{array}{l}\text { Non- } \\
\text { responders } \\
\text { migraine } \\
(n=7)\end{array}$ \\
\hline $\begin{array}{l}\text { Length of history (years M } \\
\text { (SD)) }\end{array}$ & $7.3(12.9)$ & $7.4(10.2)$ \\
\hline Intensity (/10; M (SD)) & $7.9(1.7)$ & $8.0(1.8)$ \\
\hline $\begin{array}{l}\text { Frequency (headache days } \\
\text { per week) }\end{array}$ & $5.9(1.6)$ & $6.3(1.2)$ \\
\hline $\begin{array}{l}\text { Onset related to neck trauma/ } \\
\text { neck pain }\end{array}$ & $50 \%$ & $14.3 \%$ \\
\hline \multicolumn{3}{|l|}{ Location } \\
\hline Bilateral & $60 \%$ & $43 \%$ \\
\hline Unilateral & $20 \%$ & $0 \%$ \\
\hline Changes sides & $20 \%$ & $57 \%$ \\
\hline Pulsating quality & $40 \%$ & $57 \%$ \\
\hline Physical activity aggravates & $75 \%$ & $43 \%$ \\
\hline $\begin{array}{l}\text { Neck movement, postures } \\
\text { aggravate }\end{array}$ & $89 \%$ & $86 \%$ \\
\hline $\begin{array}{l}\text { Headache brought on by } \\
\text { pressure to back of the head/ } \\
\text { neck }\end{array}$ & $100 \%$ & $43 \%$ \\
\hline $\begin{array}{l}\text { Photophobia and } \\
\text { phonophobia }\end{array}$ & $80 \%$ & $100 \%$ \\
\hline Nausea-vomiting & $80 \%-30 \%$ & $86 \%-43 \%$ \\
\hline Premonitory symptoms & $50 \%$ & $86 \%$ \\
\hline
\end{tabular}

from the cervical blocks. Thus, for the majority of participants who were non-responders, the headache and neck pain were unlikely to be related to a cervical disorder with neck pain being more likely a headache symptom reflecting the interaction between trigeminal and cervical afferents and the bidirectional pathway in the trigeminocervical nucleus. ${ }^{6}$ This clearly illustrates the need for a comprehensive physical examination of the cervical region to be part of the differential diagnostic process to confirm or dismiss a cervical source of headache and neck pain. This study found that accurate differential diagnosis of cervicogenic headache can only be made if the physical examination reveals a pattern of cervical musculoskeletal impairments of at least, reduced movement, symptomatic joint dysfunction and impaired muscle function. Single signs of neck dysfunction or tenderness in this study failed to predict responsiveness to cervical diagnostic blocks.

Confirmation of the validity of the pattern of physical signs to accurately recognise a cervical origin of neck pain in headache stands to strengthen current diagnostic criteria for cervicogenic headache. ${ }^{313}$ With the well-known symptomatic overlap between recurrent headache types, a requirement for the presence of the pattern of cervical physical signs could lessen the incidence of misdiagnosed 
migraine, ${ }^{9}$ misdiagnosed cervicogenic headache ${ }^{10}$ and promote early suitable patient management.

\section{Limitations}

Our sample was relatively small, but recruiting 381 participants was not feasible given the interventional nature of the gold standard reference diagnosis. We therefore used LASSO for a more robust evaluation of our hypothesis. Even with the small sample, the results confirmed findings from previous studies that the full pattern of cervical dysfunction is necessary to accurately diagnose cervical dysfunction as the source of neck pain in participants with recurrent headaches. ${ }^{1517}$

Diagnostic blocks were limited to the C2/C3 and C3/ $\mathrm{C} 4$ facet joints, even though the $\mathrm{C} 0 / \mathrm{C} 1$ and $\mathrm{C} 1 / \mathrm{C} 2$ articulations can cause headache. ${ }^{34}$ To account for this limitation, positive symptomatic joint dysfunction was defined so that 'C2/C3-C3/C4 joint dysfunction' represented signs at these joints that were equal to or greater than signs at higher levels. Furthermore, the FRT was added to the clinical examination as an additional cervical sign to account for headache stemming from $\mathrm{C} 1 / \mathrm{C} 2$. This decision was appropriate because the FRT was retained in the model and greater FRT values (ie, less likely C1/C2 dysfunction) were associated with greater chance of relief with C2/C3 and C3/C4 anaesthetic blocks (table 2).

Lastly, greater occipital nerve blocks are used in the management of migraine, ${ }^{47}$ as this nerve arises from the C2 and C3 dorsal rami, it introduces the possibility that the TON and the $\mathrm{C} 3 / \mathrm{C} 4$ medial branch blocks may provide a similar effect on headache without reference to cervical dysfunction. This is less likely as the greater occipital nerve has multiple innervations and in headache, is frequently thought to contribute to neck pain via sensitised trigeminal fibres, whereas the controlled diagnostic blocks performed in this study are specific to the C2/C3 and C3/C4 facet joints. As well, responders to the blocks consistently presented with the pattern of cervical dysfunction characteristic of a musculoskeletal disorder. Furthermore, a recent systemic review found no strong evidence for the presence of cervical musculoskeletal dysfunction in migraine to account for the neck pain. ${ }^{48}$

\section{CONCLUSION}

This study confirmed, against the gold standard of controlled diagnostic blocks, that findings of a pattern of cervical musculoskeletal dysfunction from a clinical examination are necessary for accurate identification of cervical related neck pain. There is now fairly strong evidence to support the importance of this pattern of cervical impairment, rather than single signs such as ROM. It has exhibited good performance in different samples with different examiners and with different reference standards. Adopting the criterion pattern of cervical musculoskeletal dysfunction could strengthen current diagnostic criteria for cervicogenic headache in a clinical examination and more effectively differentially diagnose the source of neck pain so commonly associated with migraine and tension-type headache.

\section{Author affiliations}

${ }^{1}$ AMITA Neuroscience Institute, AMITA Health Saint Joseph Medical Center, Joliet,

Illinois, USA

${ }^{2}$ College of Health Sciences, University of Saint Augustine for Health Sciences, Saint Augustine, Florida, USA

${ }^{3}$ AMITA Pain Care, AMITA Health Saint Joseph Medical Center, Joliet, Illinois, USA

${ }^{4}$ School of Physical Therapy and Athletic Training, Pacific University, Hillsboro, Oregon, USA

${ }^{5}$ Centre of Clinical Research Excellence in Spinal Pain, Injury and Health, The University of Queensland, Brisbane, Queensland, Australia

Acknowledgements The authors would like to thank Geovanni Berardi for his assistance with data management, neurologist Marquess Wilson, anaesthesiologists Asad Cheema and Udit Patel, neurosurgeons Tamir Hersonskey and Beejal Amin and nurse practitioners Kelly Morris, Sheryl Burcenski and Colleen Grandowski for participant recruitment.

Contributors SLG made substantial contributions to the conception and design of the study, the analysis and interpretation of the data, and drafting/revision of the manuscript. SMG made substantial contributions to the acquisition of data regarding the neurology diagnosis and drafting/revision of the manuscript. I0 made contributions to the acquisition of data regarding the anaesthetic blocks and drafting/revision of the manuscript. RJN made substantial contributions to the analysis and interpretation of data and drafting/revision of the manuscript. GJ made substantial contributions to the design of the study, the analysis and interpretation of the data, and drafting/revision of the manuscript. All authors read and approved the final manuscript. All authors agreed to be accountable for both personal contributions as well as the manuscript as a whole.

Funding The authors have not declared a specific grant for this research from any funding agency in the public, commercial or not-for-profit sectors.

Competing interests None declared.

Patient consent for publication Not required.

Ethics approval Ethical approval was granted by the Institutional Review Board at Presence St. Joseph Medical Center in Joliet, Illinois (00005323). All procedures were conducted in accordance with the Declaration of Helsinki.

Provenance and peer review Not commissioned; externally peer reviewed.

Data availability statement Data and full protocol are available upon request to the corresponding author.

Open access This is an open access article distributed in accordance with the Creative Commons Attribution Non Commercial (CC BY-NC 4.0) license, which permits others to distribute, remix, adapt, build upon this work non-commercially, and license their derivative works on different terms, provided the original work is properly cited, appropriate credit is given, any changes made indicated, and the use is non-commercial. See: http://creativecommons.org/licenses/by-nc/4.0/.

ORCID iD

Scott L Getsoian http://orcid.org/0000-0002-0333-7501

\section{REFERENCES}

1 Blumenfeld A, Siavoshi S. The challenges of cervicogenic headache. Curr Pain Headache Rep 2018;22:47.

2 Kaniecki RG. Migraine and tension-type headache: an assessment of challenges in diagnosis. Neurology 2002;58:S15-20.

3 Headache Classification Committee of the International Headache Society. The International classification of headache disorders, 3rd edition. Cephalalgia 2018;38:1-211.

4 Ashina S, Bendtsen L, Lyngberg AC, et al. Prevalence of neck pain in migraine and tension-type headache: a population study. Cephalalgia 2015;35:211-9.

5 Lampl C, Rudolph M, Deligianni $\mathrm{Cl}$, et al. Neck pain in episodic migraine: premonitory symptom or part of the attack? $\mathrm{J}$ Headache Pain 2015;16:80.

6 Bartsch T. Migraine and the neck: new insights from basic data. Curr Pain Headache Rep 2005;9:191-6. 
7 Bartsch T, Goadsby PJ. Increased responses in trigeminocervical nociceptive neurons to cervical input after stimulation of the dura mater. Brain 2003;126:1801-13.

8 Bartsch T, Goadsby PJ. Stimulation of the greater occipital nerve induces increased central excitability of dural afferent input. Brain 2002;125:1496-509.

9 Viana M, Sances G, Terrazzino S, et al. When cervical pain is actually migraine: an observational study in 207 patients. Cephalalgia 2018;38:383-8.

$10 \mathrm{Yi} \mathrm{X,} \mathrm{Cook} \mathrm{AJ,} \mathrm{Hamill-Ruth} \mathrm{RJ,} \mathrm{et} \mathrm{al.} \mathrm{Cervicogenic} \mathrm{headache} \mathrm{in}$ patients with presumed migraine: missed diagnosis or misdiagnosis? $J$ Pain 2005;6:700-3.

11 Rota E, Zucco R, Guerzoni S, et al. Migraine awareness in Italy and the myth of "cervical srthrosis". Headache 2020;60:81-89.

12 Calhoun AH, Ford S, Pruitt AP. Presence of neck pain may delay migraine treatment. Postgrad Med 2011;123:163-8.

13 Sjaastad O, Fredriksen TA, Pfaffenrath V. Cervicogenic headache: diagnostic criteria. Headache 1998;38:442-5.

14 Stenneberg MS, Rood M, de Bie R, et al. To what degree does active cervical range of motion differ between patients with neck pain, patients with whiplash, and those without neck pain? A systematic review and meta-analysis. Arch Phys Med Rehabil 2017;98:1407-34.

15 Jull G, Amiri M, Bullock-Saxton J, et al. Cervical musculoskeletal impairment in frequent intermittent headache. Part 1: subjects with single headaches. Cephalalgia 2007;27:793-802.

16 Malmström E-M, Karlberg M, Fransson PA, et al. Primary and coupled cervical movements: the effect of age, gender, and body mass index. A 3-dimensional movement analysis of a population without symptoms of neck disorders. Spine 2006;31:E44-50.

17 Amiri M, Jull G, Bullock-Saxton J, et al. Cervical musculoskeletal impairment in frequent intermittent headache. Part 2: subjects with concurrent headache types. Cephalalgia 2007;27:891-8.

18 Edlow BL, Wainger BJ, Frosch MP, et al. Posterior circulation stroke after $\mathrm{C} 1-\mathrm{C} 2$ intraarticular facet steroid injection: evidence for diffuse microvascular injury. Anesthesiology 2010;112:1532-5.

19 Takasaki H, Hall T, Oshiro S, et al. Normal kinematics of the upper cervical spine during the flexion-rotation test - In vivo measurements using magnetic resonance imaging. Man Ther 2011;16:167-71.

20 Hall TM, Robinson KW, Fujinawa O, et al. Intertester reliability and diagnostic validity of the cervical flexion-rotation test. J Manipulative Physiol Ther 2008;31:293-300.

21 Hall TM, Briffa K, Hopper D, et al. Comparative analysis and diagnostic accuracy of the cervical flexion-rotation test. $J$ Headache Pain 2010;11:391-7.

22 Youdas JW, Carey JR, Garrett TR. Reliability of measurements of cervical spine range of motion--comparison of three methods. Phys Ther 1991;71:98-104.

23 Tousignant M, de Bellefeuille L, O'Donoughue S, et al. Criterion validity of the cervical range of motion (CROM) goniometer for cervical flexion and extension. Spine 2000;25:324-30.

24 de Koning CHP, van den Heuvel SP, Staal JB, et al. Clinimetric evaluation of active range of motion measures in patients with nonspecific neck pain: a systematic review. Eur Spine J 2008;17:905-21.

25 Fletcher JP, Bandy WD. Intrarater reliability of CROM measurement of cervical spine active range of motion in persons with and without neck pain. J Orthop Sports Phys Ther 2008;38:640-5.

26 Falla DL, Jull GA, Hodges PW. Patients with neck pain demonstrate reduced electromyographic activity of the deep cervical flexor muscles during performance of the craniocervical flexion test. Spine 2004;29:2108-14.

27 Juul T, Langberg $\mathrm{H}$, Enoch $\mathrm{F}$, et al. The intra- and inter-rater reliability of five clinical muscle performance tests in patients with and without neck pain. BMC Musculoskelet Disord 2013;14:339.

28 Jull G, Bogduk N, Marsland A. The accuracy of manual diagnosis for cervical zygapophysial joint pain syndromes. Med J Aust 1988;148:233-6.
29 Schneider GM, Jull G, Thomas K, et al. Intrarater and interrater reliability of select clinical tests in patients referred for diagnostic facet joint blocks in the cervical spine. Arch Phys Med Rehabil 2013;94:1628-34.

30 Narouze S, Benzon HT, Provenzano D, et al. Interventional spine and pain procedures in patients on antiplatelet and anticoagulant medications (second edition): guidelines from the american society of regional anesthesia and pain medicine, the european society of regional anaesthesia and pain therapy, the american academy of pain medicine, the international neuromodulation society, the north american neuromodulation society, and the world institute of pain. Reg Anesth Pain Med 2018;43:225-62.

31 Boswell MV, Singh V, Staats PS, et al. Accuracy of precision diagnostic blocks in the diagnosis of chronic spinal pain of facet or zygapophysial joint origin. Pain Physician 2003;6:449-56.

32 Cohen SP, Stojanovic MP, Crooks M, et al. Lumbar zygapophysia (facet) joint radiofrequency denervation success as a function of pain relief during diagnostic medial branch blocks: a multicenter analysis. Spine J 2008;8:498-504.

33 Manchikanti L, Abdi S, Atluri S, et al. An update of comprehensive evidence-based guidelines for interventional techniques in chronic spinal pain. Part II: guidance and recommendations. Pain Physician 2013;16:S49-283.

34 Bogduk N. Anatomy and physiology of headache. Biomed Pharmacother 1995;49:435-45

35 Rathmell JT. Atlas of image guided intervention in regional anesthesiology and pain medicine. 2nd ed. Philadelphia, PA Lipincott Williams \& Wilkins, 2012.

36 Bogduk N. International spinal injection society guidelines for the performance of spinal injection procedures. Part 1: zygapophysial joint blocks. Clin J Pain 1997;13:292-7.

37 Ogundimu EO, Altman DG, Collins GS. Adequate sample size for developing prediction models is not simply related to events per variable. J Clin Epidemiol 2016;76:175-82.

38 Sjaastad O, Bakketeig LS. Prevalence of cervicogenic headache: Vågå study of headache epidemiology. Acta Neurol Scand 2008;117:173-80.

39 Tibshirani R. Regression shrinkage and selection via the LASSO Journal of the Royal Statistical Society: Series B 1996;58:267-88.

40 Efron B. Estimating the error rate of a prediction rule: improvement on cross-validation. J Am Stat Assoc 1983;78:316-31.

41 Hartmann A, Van Der Kooij AJ, Zeeck A. Exploring nonlinear relations: models of clinical decision making by regression with optimal scaling. Psychother Res 2009;19:482-92.

42 Steyerberg EW, Vickers AJ, Cook NR, et al. Assessing the performance of prediction models: a framework for traditional and novel measures. Epidemiology 2010;21:128-38.

43 Allison PD. Convergence failures in logistic regression. SAS Global Forum 2008;360:1-11.

44 Royston P, Altman DG. Visualizing and assessing discrimination in the logistic regression model. Stat Med 2010;29:2508-20.

45 Hastie T, Tibshirani R, Friedman J. The elements of statistical learning: data mining, inference, and prediction. Springer New York: Springer Series in Statistics, 2009.

46 Dodick D, Silberstein S. Central sensitization theory of migraine: clinical implications. Headache 2006;46 Suppl 4:S182-91.

47 Inan LE, Inan N, Karadaș Ö, et al. Greater occipital nerve blockade for the treatment of chronic migraine: a randomized, multicenter, double-blind, and placebo-controlled study. Acta Neurol Scand 2015;132:270-7.

48 Liang Z, Galea O, Thomas L, et al. Cervical musculoskeletal impairments in migraine and tension type headache: a systematic review and meta-analysis. Musculoskelet Sci Pract 2019;42:67-83. 